\title{
Original-Mittheilung.
}

Neue Standorte:

1. Dianthus superbus L. bei Wilhelmsdorf! (Ullepitsch), Haschendorf (L. Richt. et Steffek).

2. Rosa spinosissima L. var. spinosa Neilr. bei Poisdorf und Wilhelmsdorf (Ullepitsch).

3-4. R. biserrata Mer. und $R$. Podolica Tratt., Haschendorf (Richt. und Steffek).

\section{Botanische Gesellschaften, Vereine, Congresse etc.}

\section{Deutsche botanische Gesellschaft in Berlin.}

Sitzung am 20. Juli 1890.

Zur Vorlage gelangte eine Abliandlung ${ }^{-1}$ ) betitelt:

\section{Versuch einer Erklärung des Wachsthums der Pflanzenzelle.}

\section{Von Prof. Dr. Julius Wiesner.}

Die meisten Botaniker nehmen an, dass das Wachsthum der Zelle und ihrer Theile durch Intussusception erfolge. Andere verwerfen die Intussusceptionslehre vollständig und versuchen, alle Vorgänge des Wachsthums der Zelle und ihrer Theile auf Apposition zurüekzuführen. Die Letzteren nehmen dabei eine alte Lehre wieder auf, bringen dieselbe aber in eine modificirte Form, indem sie namentlich starke, nachträgliche Dehnungen der durch Apposition entstandenen Theile nachzuweisen suchen, um deren Flächenwachsthum verständlich zu machen. Aber weder die Intussusceptions- noch die Appositionstheorie bat zu einer befriedigenden Lösung der Wachsthumsfrage geführt und auch der Versuch, einzelne Vorgänge durch Intussusception, andere durch Apposition zu erklären, kann nicht als gelungen betrachtet werden.

Auf eine Kritik all' dieser Theorien brauche ich umso weniger einzngehen, als ich es unternehme, aus neuen Gesichtspuukten eine Erklärung des Wachsthums der Zelle zn versuchen.

Ich gehe bei diesem meinen Versuche von dem uns sehr anschaulich vorliegenden Wachsthum eines vielzelligen Pflanzentheiles, zum Beispiel eines Blattes oder Stammes, aus. Sehen wir von dem Wachsthum der einzelnen Zellen eines solchen Organs ab, so vollzieht sich das Wachsthum des letzteren derart, dass die einzelnen Zellen sich ausdehnen, nachdern der Vorgang der Volumsvergrösserung durch Theilung eingeleitet wurde. Die Theilung der Zellen führt selbst noch nicht zum Wachsthum des Organs, ist aber für dieses

1) Vergl. Berichte der deutschen botan. Gesellsch., VIII., S. 196.

Oesterr. hotan. Zeit sehrift. $\mathrm{J}_{0}$. Heft 1890 . 
unbedingt nothwendig. Die neu entstandenen Zellen dehnen sich ans, gleichmässig oder ungleichmässig, und durch das Zusammenwirken der ihr Volumen vergrössernden Zellen gewinnt das Organ seine Gestalt. vergrössert seine Oberfläche, wächst in die Dicke, Länge und Breite, krümmt sich häufig in Folge urigleichmässigen Wachsthums der einzelnen Elemente oder der Zellschichten u. s. w. Immer aber erfolgt die Neubildung und Vergrösserung der Theile zwischen Grenzen: intercalar, ohne dass etwas von aussen zugefügt, oder dass ein Nenes von aussen eingefügt werden würde.

Indem man das Wachsthum der Zelle und ihrer Theile verfolgt, entrollt sich uns ein selnr ähnliches Bild. Denn auch hier sehen wir ohne sichtliche Einschiebung Oberflächenvergrösserung, Dicken- und Längenzunahme, Gestaltänderung in Folge ungleichmässigen Wachsthums u. s. w. Das Wesentliche des Wachsthums der Zellentheile ist aber der intercalare Charakter aller organischen Neubildungen. Gerade dieser augenfällige Wachsthumsmodus hat ja auf die Ideo der Intussusception geführt.

Die Aehnlichkeit zwischen den Wachsthumsvorgängen dor aus Zellen aufgebauten Organe und den Wachsthumsvorgängen der Zellen ist aber nicht blos eine äusserliche. Die nachfolgende Erörterung wird zeigen, dass auch innere Gründe für eine solche Uebereinstimnung sprechen, ja dass wir unter bestimmten Voraussetzungen geradezu gezwungen sind, die Analogie dieser beiden Vorgänge einzuräumen.

Gibt es innerhalh des Organismus eine Erzeugung lebender Substanz aus todtem Stoffe? Mit anderen Worten: Kann ein lebender, der Zelle angeböriger Körper aus todter Materie entstehen? z. B. ein Zellkern, ein Chlorophyllkorn ans chemischen Individuen, aus Zucker, Chlorophyll, Fett, Eiweiss etc.? Alle unsere Erfahrungen sprechen gegen eine Bejahung dieser Frage. Es sind ja geradezu alle Angaben über eine spontane Erzeugung organisirter Gebilde innerhalb der Zellen vollständig widerlegt worden. Wenn die gestellten Fragen müssig erscheinen, da ihre Beantwortung etwas beinahe Selbstverständliches bedeutet, so kann mir das nur willkommen sein, denn die Behauptung, innerhalb des Organismus gehe Lebendes nur aus Lebendem ${ }^{1}$ ), Organisirtes nur aus Organisirtem hervor, bildet die erste meiner Voraussetzungen.

Die Form, welche ich meiner Behauptung gegeben habe, zeigt, dass ich die "Organisation" als eine specifische Structur ansehe, welche nur der" lebenden Substanz, dem Protoplasma, zukommt und welche sich keineswegs mit der Molecularst uctur deckt. Die chemischen Individnen, welche in den Aufbau der Zellen eintreten, besitzen

1) Um nicht nllzugrosser Kürze halber missverstanden zu werden, bemerke ich, dass bei der Assimilation - die ich hier und stets im weitesten Sinne nehme - selbstverständlich todte Substanzen (Nahrung) in' Bestandtheile der Gewebe, und häufig in lebende Bestandtheilo desselben umgewandelt werden; es geschieht dies aber doch nur bei Gegenwart, ja unter unbedingt nothwendiger Mitwirkung des Lebenden. 
Molecularstructur, allein die Zusammenfügung der leblosen Theile auch zu dem elementarsten lebenden Gebilde ist eine specifische. Dieser Scheidung des Lebenden von dem Unorganischen aus morphologischem Gesichtspunkte stimmen die uneisten Naturforscher zu. Andere werden die hier gezogene Grenze nicht gelten lassen; diesen gegenüber sind aber meine Argumente ohnedies wirkungslos.

Die z weite Voraussetzung, welche ich machen muss, ist die, dass kein anderer Modus der Neubildung im Organismus stattfindet, als der der Theilung. ") Alle Neubildungen des Organismus sind Zellen, oder Zellencomplexe oder endlich Theile von Zellen. Die Zelle geht aber aus der Zelle, in letzter Linie wenigstens, durch Theilung hervor, und auch die lebenden Theile der Zelle entstehen durch Theilung: der Kern aus dem Kern, das Chlorophyllkorn aus dem Chlorophyllkorn oder aus Chlorophyllkorn-Anlagen u. s. w. Die Tbeilung ist mit dem Werden aller Lebewesen so verknüpft, dass die Annahme, sie spiele vielfach auch dort eine Rolle, wo wir mit unseren beschränkten Mitteln ihr $\theta$ Wirksamkeit noch nicht erweisen können, wohlberechtigt erscheineu dürfte. ${ }^{2}$ ) Existirt überhaupt noch ein anderer Modus der Neubildung im Organismus, als der durch Theilung? Will man den Boden der Thatsachen nicht verlassen, so gibt es auf diese Frage keine andere Antwort als: nein. Und fragt man sich, ob noch eine andere Art der Neubildung des Lebenden ausser der Theilung denkbar ist, so kommt man nur auf den Ausweg, eine Erzeugung des Lebenden aus dem Leblosen anzunehmen, woraus zu ersehen ist, wie innig meine zweite Voraussetzung mit der ersten verknüpft ist.

Räumt man die beiden gemachten Voraussetzungen ein - nnd die meisten Botaniker werden beide als selbstverständliche betrachten - so ergibt sich mit logischer Nothwendigkeit, dass das (schou sichtlich complex gebaute) Protoplasma sich obne jnuere Theilung njcht regeneriren könne. ${ }^{3}$ ) Wenn also eine Meristemzelle sich mehrmals getheilt hat und dabei selbstverständlich ihre lebende Substanz sich vermehrte, so ist diese Neubildung des Protoplasmas auf Grund innerer Theilung erfolgt.

Dieser Argumentation zufolge muss die lebende Substanz der

1) Die Coujugation ridorspicht obigem Satze nicht, da diesclbe doch

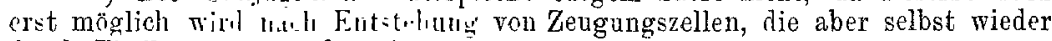
durch Theilung entstanden sind.

2) Die Geschichte der Zellenlehre gibt uns whl zu verstehen, dass wir eine vollständige Kenntniss der Verbreitung der Theilningsvorgänge noch kaum besitzen dürften. da ron Jahr zu Jahr neue Formen der Theilung und neue theilungsähige Gebilde entdeckt werden. Es ist ja gar noch nicht so lange her, dass inan anch eine nicht auf Theilung berulende Zellenvermehrung annabm, Kern und Chlorophlibirner apnntan im Protoplasma entstehen liess a. s. w.

$\left.{ }^{3}\right)$ Von dirs. illuen Th-ilnlle ist nur wenig direct durch Beobachtong zu constativen: li Theilung korn-Anlagen un alible Grbili: der Meristemzellen. Dass aber innerhalb dieser sich theilenden Bestandtheile der Zelle noch Theilungen stattfinden, lassen schon dic karyokinetischen Erscheinungen annehmen. 
Pflanze - und zu dieser rechne ich nicht nur das Protoplasma im engeren Sinne (Cytoplasma) und Kern, sondern auch die wachsende Zellhant") - aus kleinen organisirten Individualitäten bestehen, welche die Eicyenschaft besitzen, sich zu theilen.

Wenn aber diese kleinsten organischen Individualitäten sich theilen, so müssen sie anch die Fäbigkeit baben zn wachsen, denn sunst müssten sie schliesslich so weit zeriallen, dass sie nicht mehr als etwas Organisirtes betrachtet werden könnten. Wenn sie aber wachsen, so müssen sie anch assimiliren.

Räumt man also ein, dass im Organismus Lebendes aus Todtem nicht hervorgehen könne und dass die Neubildung stets auf Theilung zurückzuführen ist, so muss man anch zugeben, dass die lebende Substanz aus kleinen organisirten Individualitäten bestehen müsse, welche die Fähigkeit habon, sich zu theilen, zu wachsen und zu assimiliren.

Ich bezeichue diese einfachsten Elementarorgane der Zelle als Plasomen. In wie weit wir derartige, der Zelle untergeordnete Elementargebilde anzunehmen berechtigt sind, darüber und übor den Versuch, dieselbe nachzuweisen, habe ich mich an anderer Stelle ansgesprocben. ${ }^{2}$ )

Dass die Plasomen unter einander verbunden sind, wie etwa die Zellen eines Gewebes, wird gewiss Jeder zugeben, der ihre Existenz überhaupt einräumt. Ihre gegenseitige Verbinang ist wahrscheinlich eine sehr verschiedenartige, doch werden sie wohl zumeist in der Weise verknüpt sein, dass sie wenigstens theilweise mit Flüssigkeiten in Berührung stehen.

Wenn aber die Zelle und ihre lebenden Theile aus Plasomen so zusammengefügt sind, wie etwa ein Blatt aus Zellen sich zusammensetzt, so muss das Wachsthum der Zolle ebenso durch das Wachsthum ihrer Plasomen erfolgen, wie ein vielzelliges Organ in Folge der organischen Volumsvergrösserung seiver Zellen wächst.

Es frägt sich nun weiter, in welcher Weise die ElementarOrgane der Zelle, die Plasomen, also dje letzten noch theilungsfähigen Elemente der Zelle, wachsen. Als Bestandtheile des Protoplasma (inclusive Ker'n und Dermatoplasma) sind sie gleich diesem weiche, plastische Massen, welchen in Folge Besitzes grosser Mengen von Wasser eine leichte Versehiebbarkeit der Theilchen zukommt. Es ist deshalb durchaus nicht nothwendig, zur Erklärung ilırer Grössen- und Inhaltszuvahme Intussusception anzınehmen. In Anbetracht der genannten physikalischen Beschaffenheit der lebenden Sulustanz ist anch schon von anderer Seite ${ }^{3}$ ) darauf hingewiesen

1) S, hierüher Wiesncr, Untersuchungen übex die Organisation der vegetabilisehen \%elthaut. Sitzungsber. d. kais. Akad. d. Wissenscbaften zu Wien, math.-nat. Classe, Bd. 93. (1886).

) S. Sitzung sanzeiger der math-nat. Cl. d. kajs. Akad. d. Wissenschaften. Wien 1890. Nr. XIII und Sitzungsber. Bd. 99 (1890).

3) Bütschli, in biol. Centralbl. vom Mai 1888, s. $161 \mathrm{ff}$. 
Worden, dass zur Erklärung des Wachsthums des Protoplasma die Annahme einer Intussusception im Sinne der herrschenden Lehre nicht erforderlich sei.

Die Volumszunahme eines eben getheilten Plasoms lässt sich einfach physikalisch erk]ären. Auf dem Wege der Diffusion und Absorption treten Wasser und gelöste feste Körper, beziehungsweise Gase in diese Körperchen ein und werden daselbst assimilirt, wobei die festen Assimilationsproducte das Volum des Plasoms fixireu. Dio Fin- und Anlagerung der zugewachsenen Substanz ist sobin physikalisch ganz gut begreiflich. Fraglich bleibt nur, wie im Plasom die eintretenden oder gebildeten chemischen Individuen, denen eine bestimmte Molecularstructur zukommt, unter dem Einfluss der lebenden Substanz organisirt werden, d. h. wie die todten Baustein $\theta$ in die schon bestehende lebende Einheit sich so einfügen, dass dieselbe uniter den Bedingungen ihrer Existenz in einem bestimmten Zeitpunkt aufgehoben wird und Theilung eintritt.

Wie das Molecül das letzte Formelement der todten Substanz jet, so bildet nach meiner Auffassung das Plasom das letzte mit den Attributen des Lebens ansgerüstete Formelement des Organismus.

Auf der Theilungsfähigkeit der Plasomen beruhen nach dieser meiner Auffassung schliesslich alle Theilungsvorgänge der Zelle: theilt sich beispielsweise das Protoplasma (in toto) oder ein Chloroplynlkorn. so ist es eine Schicht von Plasomen, in welchen die Theilung sich vollzieht.

Das Wachsthum des Protoplasma ist von dem Wachsthum der Plasomen wohl zu unterscheiden. Das Plasom ergänzt blos durch das Wachsthum seine Masse, das Protoplasma wächst durch Neubildung von wachsenden Plasomen.

Wenn auch, nach meiner Aliffassung, das Wachsthum der Zelle auf dem Wachisthum seiner Plasomen beruht, so ist doch nicht ausgeschlossen. dass auch Dehnungen bei der Volumsvergrösserung der Zelle betheiligt sind und zwar in gleichem Sinne, wie beim Wachsthum eines aus Zellen bestehenden Organs. Bejspielsweise sehen wir die Oberhant häufig eine Oberfläche erreichen, welche nicht allein aus dem Wachsthum ibrer Zellen resultirt, sondern zum Theil auf dem dehnenden Drucke beruht, den das Parenchym auf das genannte Gewebe ausübt. So gebe ich auch Dehnungen der Zellhaut zu, hervorgerufen durch den Gesammtdruck des Cytoplasma.

Eine ausführliche Begrïndung und Erläuterung der hier blos aphoristisch dargestellten Anschanungen werde jch später an anderer Stelle veröffentlichen.

Dı ich aber durch jahrelange diesbezügliche Studien zu Resultaten gelangte, welche sich in Kürze wiedergeben lassen und ich vielleicht erst nach längerer Zeit die ausführliche Abhandlung werde veröffentlichen kömnen, so habe ich es unternommen, meinen bescheidenen Versuch, die Wachsthumsfrage von neuen Gesichtspunkten 
aus zu betrachten, den Fachgenossen schon in der vorliegenden Form bekanut zu geben.

Ich hoffe, man wird in diesem meinem Unternehmen, eine der Grundfragen des Lebens zu erörtern, nicht die Sucht nach einer Neuerung erblicken. Vielmehr darf ich erwarten, dass die unbefangenen Prüfer der hier vorgetragenen Auffassungen in diesem meinem Versuche das Bestreben erkennen werden, jene Punkte ansfindig zu machen, nach welchen hin die neuen Resultate über Leben und Entwickelung der Zelle, und namentlich über die Bedeutung der Theilungsvorgänge der Zelle convergiren.

\section{Botanische Forschungsreisen,}

Aus Briefen des zur Zeit auf einer zweiten kleinasiatischen Reise befindlichen Herrn J. Bornmüller ist ersichtlich, dass derselbe auch diesmal sehr reiche und interessante Sammlungen angelegt hat. Am Anfang des voligen Monats schrieb er, dass er nach einer fünfwöchentlichen Rurdtour von Amasia über Siwas, Kaisar ieth. Jusgat wieder wohlbehalten in ersterer Stadt eingetroffen ist. Bei Siwas fand er auf dem 1800-2000 M. hohen Tschamlübel-dagh und auf dem benachbarten $2520 \mathrm{M}$. hohen Yildiz-dagh eine ron dem Amasia-Gebiet ganz abweichende Flora. Die Ersteigung des spitzen Kegels des letzteren machte ein zwölfstündiges Aufsitzen nöthig. Anf dem Plateau war Gymnandra stolonifera sebr häufig, Torfwiesen waren oft von Primula auriculata blau, Felswände von Papaver orientale roth gefärbt. Der steile Geröllkegel barg Massen von Pulsatilla Armena, Ranunculus anemonifolius, Geranium subcaulescens, sowie prächtige Fritillarien. Bei Siwas durchschritt er, nicht obne Gefahr für sein Gepäck, unter strömenden Regengüssen den Halys, dessen Berglehnen in Menge mit einem azurblanen Paracaryum bestanden waren, während die Felder zahlreiches Verbascum Wiedemannianum, Gypsophila Wiedemanniana, Centaurea depressa, Bupleurum crocenm, Wiedemannia multifida u. v. a. bcherbergten. Beim ersten Erblicken des M. Argaeus war er höchst überrascht, denn einen solchen Schneecoloss, der wie aus den Wolken hervorgezaubert vor $\mathrm{ihm}$ stand, hatte er nicht erwartet. Die Ersteigung nabm vier Tage in Anspruch; die letzten 1500 M. nichts als Schnee und Gletscher, über die er nur von der Südseite her auf einem Geröllrücken gelaugen konnte. Nur die letzten 150 des 13000 Fuss hohen Berges waren unersteigbar, eine wild zerrissene Felsenkrone, ewigen Winter bildend. Schon bei 2900 M. war von Baumwuchs keine Spur mehr zu selıen, nur vereinzelt kommt noch kümmerlicher Ardudsch (Juniperus nana) in den Felsen vor, der nebst Traganth-Sträucheru das einzige Brennmaterial bildet. Drei Nächte unter freiem Himmol obne Fener bei $+2-3^{\circ}$ R. in jener Region zu verbriugen, waren jlim nicht angenehm, umsomehr, da die Vegetation noch sebr zurück war. Arn Fusse sammelte er die schöne Cousinia Caesarea 